\title{
Basic concepts and equations of solid mechanics
}

\author{
Milan Jirásek \\ Department of Mechanics \\ Faculty of Civil Engineering \\ Czech Technical University in Prague \\ 16629 Prague, Czech Republic \\ Milan.Jirasek@epfl.ch
}

\begin{abstract}
This paper provides a brief overview of the basic concepts and equations that will be used by other papers in the special issue on Damage and Fracture in Geomaterials. The first section introduces the tensorial notation, defines the basic tensor operations and presents the formalism for the volumetric-deviatoric decomposition of stress and strain. The second section shows how to transcribe tensorial relations in the engineering notation. The basic equations describing a linear elastic deformable body are summarized in the third section, and their discretization by the finite element method is described in the fourth section, with an extension to nonlinear constitutive relations in the fifth section. The last section outlines a thermodynamic framework based on two potentials-the free energy and the dissipation potential.

RÉSUMÉ. Cet article présente une introduction sur les idées de base et les équations qui sont utilisées dans cet ouvrage sur l'endommagement et la rupture des géomatériaux. La première partie présente les notations tensorielles, les opérations tensorielles usuelles et le concept de décomposition des contraintes et des déformations en une partie volumique et une partie déviatorique. La deuxième partie montre comment transformer ces relations tensorielles en des notations usuelles pour l'ingénieur. Les équations de bases décrivant la déformation d'un milieu élastique et leurs discrétisations par la méthode des éléments finis sont respectivement introduites dans les troisième et quatrième parties avec une extension pour les comportements non linéaires dans la cinquième partie. La dernière partie est dédiée au cadre thermodynamique basé sur deux potentiels, l'énergie libre et le potentiel dissipatif.

KEYWORDS: tensors, elasticity, finite element method, thermodynamic framework.

MOTS-CLÉS : tenseurs, élasticité, méthode des éléments finis, cadre thermodynamique.
\end{abstract}

REGC - 11/2007. Damage and fracture in geomaterials, pages 879 à 892 


\section{Tensorial notation}

Variables used in engineering mechanics usually have the character of tensors. In general, we denote first-order tensors (vectors) by lower-case Latin letters, secondorder tensors by lower-case Greek or Latin letters, and fourth-order tensors by capital Latin letters. Bold face letters represent the entire tensors (compact notation). When referring to the Cartesian components of tensors (indicial notation) we use lower-case Latin subscripts $i, j, k, l, \ldots$, which can take values 1,2 , and 3 corresponding to the Cartesian coordinate axes $x_{1}, x_{2}$, and $x_{3}$. For example, $\boldsymbol{u}$ is the displacement vector (first-order tensor) with components $u_{i}, i=1,2,3 ; \varepsilon$ is the strain tensor (secondorder) with components $\varepsilon_{i j}, i=1,2,3$, and $j=1,2,3 ; \boldsymbol{\sigma}$ is the stress tensor (secondorder) with components $\sigma_{i j}, i=1,2,3$, and $j=1,2,3$; and $\boldsymbol{D}$ is the stiffness tensor (fourth-order) with components $D_{i j k l}$, all subscripts running again from 1 to 3 . When the tensor itself has a subscript, we raise it to a superscript in the indicial notation in order to avoid confusion with the subscripts referring to individual components. For example, the components of the elastic stiffness tensor $\boldsymbol{D}_{e}$ are denoted as $D_{i j k l}^{e}$.

The transpose $\varepsilon^{T}$ of a second-order tensor $\varepsilon$ is obtained by switching the order of subscripts, i.e., $\left(\varepsilon^{T}\right)_{i j}=\varepsilon_{j i}$. If the transposed tensor is equal to the original one $\left(\varepsilon_{i j}=\varepsilon_{j i}\right.$ for all $i$ and $j$ ), it is said to be symmetric. Fourth-order tensors can exhibit minor symmetry $\left(D_{i j k l}=D_{j i k l}=D_{i j l k}=D_{j i l k}\right.$ for all $\left.i, j, k, l\right)$, or major symmetry $\left(D_{i j k l}=D_{k l i j}\right.$ for all $\left.i, j, k, l\right)$.

In expressions involving components of tensors we adhere to so-called Einstein summation convention. Repeated subscripts in a product-like expression imply summation from 1 to 3; e.g., $a_{i k} b_{k j}$ means in the "full" notation $\sum_{k=1}^{3} a_{i k} b_{k j}$.

The basic operations we need are:

- the dot product (contraction) of two first-order tensors, $\boldsymbol{u} \cdot \boldsymbol{v}=u_{i} v_{i}$, which produces a scalar, and therefore is sometimes called the scalar product,

- the double-dot product (double contraction) of two second-order tensors, $\boldsymbol{\sigma}: \varepsilon=\sigma_{i j} \varepsilon_{i j}$, which also produces a scalar, and therefore could be called the scalar product, too,

- the dot product (single contraction) of two second-order tensors, $\boldsymbol{a} \cdot \boldsymbol{b}$, which produces a second-order tensor with components $(\boldsymbol{a} \cdot \boldsymbol{b})_{i j}=a_{i k} b_{k j}$,

- the dot products (contractions) of a second-order tensor with a first-order tensor, $\boldsymbol{\sigma} \cdot \boldsymbol{n}$, or $\boldsymbol{n} \cdot \boldsymbol{\sigma}$, which produce a first-order tensor with components $(\boldsymbol{\sigma} \cdot \boldsymbol{n})_{i}=\sigma_{i j} n_{j}$, or $(\boldsymbol{n} \cdot \boldsymbol{\sigma})_{i}=n_{j} \sigma_{j i}$,

- the double-dot products (double contractions) of a fourth-order tensor with a second-order tensor, $D: \varepsilon$, or $\varepsilon: D$, which produce a second-order tensor with components $(\boldsymbol{D}: \boldsymbol{\varepsilon})_{i j}=D_{i j k l} \varepsilon_{k l}$, or $(\varepsilon: \boldsymbol{D})_{i j}=\varepsilon_{k l} D_{k l i j}$, and

- the direct product of two second-order tensors, $\boldsymbol{f} \otimes \boldsymbol{g}$, which produces a fourthorder tensor with components $(\boldsymbol{f} \otimes \boldsymbol{g})_{i j k l}=f_{i j} g_{k l}$. 
The scalar products are commutative $(\boldsymbol{u} \cdot \boldsymbol{v}=\boldsymbol{v} \cdot \boldsymbol{u}$ and $\boldsymbol{\sigma}: \varepsilon=\varepsilon: \boldsymbol{\sigma})$, the third operation is commutative if the second-order tensor is symmetric $(\boldsymbol{\sigma} \cdot \boldsymbol{n}=\boldsymbol{n} \cdot \boldsymbol{\sigma}$ if $\sigma_{i j}=\sigma_{j i}$ for all $i, j$ ), the fourth operation is commutative if the fourth-order tensor exhibits major symmetry ( $\boldsymbol{D}: \varepsilon=\varepsilon: \boldsymbol{D}$ if $D_{i j k l}=D_{k l i j}$ for all $i, j, k, l$ ), and the direct product is in general noncommutative.

An important example of a second-order tensor is the Kronecker delta, $\delta$, with components $\delta_{i j}=1$ if $i=j$ and $\delta_{i j}=0$ if $i \neq j$. It plays the role of the unit element with respect to the (single) dot product of two second order tensors, and therefore it is also called the unit second-order tensor. Let us also introduce the unit fourth-order tensor, $\boldsymbol{I}$, with components $I_{i j k l}=\delta_{i k} \delta_{j l}$. This tensor exhibits major symmetry but not minor symmetry, and it has the important property that $I: \varepsilon=\varepsilon: I=\varepsilon$ for any second-order tensor $\varepsilon$. Sometimes it is useful to work with the symmetrized unit fourth-order tensor, $\boldsymbol{I}_{S}$, which has components $I_{i j k l}^{S}=\left(\delta_{i k} \delta_{j l}+\delta_{i l} \delta_{j k}\right) / 2$. This tensor exhibits minor and major symmetry but the identity $\boldsymbol{I}_{S}: \varepsilon=\varepsilon: \boldsymbol{I}_{S}=\varepsilon$ holds only if the second-order tensor $\varepsilon$ is symmetric.

Finally, let us introduce the notation $\partial \boldsymbol{u} / \partial \boldsymbol{x}$ for the tensor produced by differentiation of a tensor-valued function $\boldsymbol{u}$ with respect to its tensorial argument $\boldsymbol{x}$. For example, if both $\boldsymbol{u}$ and $\boldsymbol{x}$ are first-order tensors then $\partial \boldsymbol{u} / \partial \boldsymbol{x}$ is a second-order tensor with components

$$
\left(\frac{\partial \boldsymbol{u}}{\partial \boldsymbol{x}}\right)_{i j}=\frac{\partial u_{i}}{\partial x_{j}}
$$

For future use, it is helpful to show the structure of the stiffness tensor in linear isotropic elasticity. In tensorial notation, the elastic stiffness is given by

$$
\boldsymbol{D}_{e}=\lambda \boldsymbol{\delta} \otimes \boldsymbol{\delta}+2 \mu \boldsymbol{I}_{S}
$$

or in subscript notation by

$$
D_{i j k l}^{e}=\lambda \delta_{i j} \delta_{k l}+\mu\left(\delta_{i k} \delta_{j l}+\delta_{i l} \delta_{j k}\right)
$$

where $\lambda$ and $\mu$ are material constants called Lamé coefficients. These coefficients are uniquely related to other pairs of material parameters characterizing a linear elastic isotropic material, such as Young's modulus $E$ and Poisson's ratio $\nu$, or bulk modulus $K$ and shear modulus $G$ :

$$
\begin{aligned}
\lambda & =\frac{E \nu}{(1+\nu)(1-2 \nu)}=K-\frac{2}{3} G \\
\mu & =\frac{E}{2(1+\nu)}=G
\end{aligned}
$$

Using [2], we can present the generalized Hooke's law as

$$
\boldsymbol{\sigma}=\boldsymbol{D}_{e}: \boldsymbol{\varepsilon}=\lambda \boldsymbol{\delta} \otimes \boldsymbol{\delta}: \boldsymbol{\varepsilon}+2 \mu \boldsymbol{I}_{S}: \boldsymbol{\varepsilon}=3 \lambda \boldsymbol{\delta} \varepsilon_{V}+2 \mu \boldsymbol{\varepsilon}
$$


where

$$
\varepsilon_{V}=\frac{1}{3} \boldsymbol{\delta}: \varepsilon
$$

is one third of the trace of the strain tensor, representing the relative change of volume. The volumetric part of the strain tensor is $\varepsilon_{V} \delta$, and when we subtract it from the strain tensor we obtain the deviatoric strain

$$
\boldsymbol{e}=\boldsymbol{\varepsilon}-\boldsymbol{\delta} \varepsilon_{V}=\varepsilon-\frac{1}{3} \boldsymbol{\delta} \otimes \boldsymbol{\delta}: \varepsilon=\left(\boldsymbol{I}_{S}-\frac{1}{3} \boldsymbol{\delta} \otimes \boldsymbol{\delta}\right): \varepsilon=\boldsymbol{I}_{D}: \varepsilon
$$

This has brought us to the deviatoric projection tensor

$$
\boldsymbol{I}_{D}=\boldsymbol{I}_{S}-\frac{1}{3} \boldsymbol{\delta} \otimes \boldsymbol{\delta}
$$

and if we introduce the volumetric projection tensor

$$
\boldsymbol{I}_{V}=\frac{1}{3} \boldsymbol{\delta} \otimes \boldsymbol{\delta}
$$

we can present the volumetric-deviatoric decomposition of the strain tensor as

$$
\begin{aligned}
\varepsilon & =\boldsymbol{I}_{S}: \varepsilon=\left(\boldsymbol{I}_{V}+\boldsymbol{I}_{D}\right): \varepsilon=\boldsymbol{I}_{V}: \varepsilon+\boldsymbol{I}_{D}: \varepsilon= \\
& =(\varepsilon)_{\mathrm{vol}}+(\varepsilon)_{\mathrm{dev}}=\varepsilon_{V} \boldsymbol{\delta}+\boldsymbol{e}
\end{aligned}
$$

The stress tensor can be decomposed in a similar way as

$$
\begin{aligned}
\boldsymbol{\sigma} & =\boldsymbol{I}_{S}: \boldsymbol{\sigma}=\left(\boldsymbol{I}_{V}+\boldsymbol{I}_{D}\right): \boldsymbol{\sigma}=\boldsymbol{I}_{V}: \boldsymbol{\sigma}+\boldsymbol{I}_{D}: \boldsymbol{\sigma}= \\
& =(\boldsymbol{\sigma})_{\mathrm{vol}}+(\boldsymbol{\sigma})_{\mathrm{dev}}=\sigma_{V} \boldsymbol{\delta}+\boldsymbol{s}
\end{aligned}
$$

where

$$
\sigma_{V}=\boldsymbol{I}_{V}: \boldsymbol{\sigma}=\frac{1}{3} \boldsymbol{\delta}: \boldsymbol{\sigma}
$$

is the mean stress and

$$
\boldsymbol{s}=\boldsymbol{I}_{D}: \boldsymbol{\sigma}=\boldsymbol{\sigma}-\sigma_{V} \boldsymbol{\delta}
$$

is the stress deviator.

The elastic stiffness tensor can also be decomposed into its volumetric and deviatoric part. Realizing that $\boldsymbol{\delta} \otimes \boldsymbol{\delta}=3 \boldsymbol{I}_{V}$, we can rewrite [2] as

$$
\begin{aligned}
\boldsymbol{D}_{e} & =\lambda \boldsymbol{\delta} \otimes \boldsymbol{\delta}+2 \mu \boldsymbol{I}_{S}=3 \lambda \boldsymbol{I}_{V}+2 \mu\left(\boldsymbol{I}_{V}+\boldsymbol{I}_{D}\right)= \\
& =(3 \lambda+2 \mu) \boldsymbol{I}_{V}+2 \mu \boldsymbol{I}_{D}=3 K \boldsymbol{I}_{V}+2 G \boldsymbol{I}_{D}
\end{aligned}
$$

because the coefficient $3 \lambda+2 \mu$ is recognized as three times the bulk modulus $K$, see relation [4], and $\mu=G=$ shear modulus. The generalized Hooke's law presented as

$$
\begin{aligned}
\boldsymbol{\sigma} & =\boldsymbol{D}_{e}: \boldsymbol{\varepsilon}=\left(3 K \boldsymbol{I}_{V}+2 G \boldsymbol{I}_{D}\right): \boldsymbol{\varepsilon}= \\
& =3 K \boldsymbol{I}_{V}: \boldsymbol{\varepsilon}+2 G \boldsymbol{I}_{D}: \boldsymbol{\varepsilon}=3 K \varepsilon_{V} \boldsymbol{\delta}+2 G \boldsymbol{e}
\end{aligned}
$$


naturally splits into the volumetric and deviatoric part:

$$
\begin{aligned}
\sigma_{V} & =3 K \varepsilon_{V} \\
s & =2 G \boldsymbol{e}
\end{aligned}
$$

\section{Engineering notation}

The tensorial notation is certainly very elegant and useful in theoretical derivations. However, when developing a numerical algorithm that should be implemented into a computer code, it is more practical to store stress and strain components in one-dimensional arrays and stiffness moduli in two-dimensional arrays. Code development is then facilitated if the basic formulas are written with stresses and strains represented by column matrices and stiffness coefficients arranged in square matrices. This is also the notation often used in engineering textbooks, and we shall call it the engineering notation (or the Voight notation).

When using the engineering notation one has to be careful about the ordering of components. The normal components are usually arranged in the natural order (i.e., $\sigma_{x}$ followed by $\sigma_{y}$ and $\sigma_{z}$ ) but for the shear components several conventions exist. In principle it is possible to use any of them, but it is extremely important to select one convention and stick to it throughout the entire project. One possibility is to set

$$
\boldsymbol{\sigma}=\left\{\begin{array}{l}
\sigma_{x} \\
\sigma_{y} \\
\sigma_{z} \\
\tau_{y z} \\
\tau_{z x} \\
\tau_{x y}
\end{array}\right\}=\left\{\begin{array}{l}
\sigma_{11} \\
\sigma_{22} \\
\sigma_{33} \\
\sigma_{23} \\
\sigma_{31} \\
\sigma_{12}
\end{array}\right\}, \quad \boldsymbol{\varepsilon}=\left\{\begin{array}{l}
\varepsilon_{x} \\
\varepsilon_{y} \\
\varepsilon_{z} \\
\gamma_{y z} \\
\gamma_{z x} \\
\gamma_{x y}
\end{array}\right\}=\left\{\begin{array}{l}
\varepsilon_{11} \\
\varepsilon_{22} \\
\varepsilon_{33} \\
\mathbf{2} \varepsilon_{23} \\
\mathbf{2} \varepsilon_{31} \\
\mathbf{2} \varepsilon_{12}
\end{array}\right\}
$$

Note that the engineering shear component $\gamma_{x y}$ is twice the tensorial shear strain $\varepsilon_{12}$, etc. The reason for introducing the factor of 2 is not only that strain component $\gamma_{x y}$ has a physical meaning of the shear angle. More importantly, we want the energy product $\sigma: \varepsilon=\sigma_{i j} \varepsilon_{i j}$ to be replaced in the engineering notation by a simple scalar product of column matrices, $\sigma^{T} \varepsilon$. Unfortunately, this simple transcription of the double contraction of two second-order tensors works only if we deal with one stress-like tensor and one strain-like tensor. When evaluating the tensorial norm of the stress tensor, defined as $\|\boldsymbol{\sigma}\|=\sqrt{\boldsymbol{\sigma}: \boldsymbol{\sigma}}$, we have to be careful and realize that

$$
\begin{aligned}
\boldsymbol{\sigma}: \boldsymbol{\sigma}=\sigma_{i j} \sigma_{i j} & =\sigma_{11}^{2}+\sigma_{12}^{2}+\sigma_{13}^{2}+\ldots+\sigma_{31}^{2}+\sigma_{32}^{2}+\sigma_{33}^{2}= \\
& =\sigma_{11}^{2}+\sigma_{22}^{2}+\sigma_{33}^{2}+2\left(\sigma_{23}^{2}+\sigma_{31}^{2}+\sigma_{12}^{2}\right)
\end{aligned}
$$

while

$$
\boldsymbol{\sigma}^{T} \boldsymbol{\sigma}=\sigma_{11}^{2}+\sigma_{22}^{2}+\sigma_{33}^{2}+\sigma_{23}^{2}+\sigma_{31}^{2}+\sigma_{12}^{2}
$$


So it is not possible to replace the tensorial norm of the stress tensor by the Euclidean norm of the column matrix of stress components! Instead, we have to insert a diagonal scaling matrix

$$
\boldsymbol{P}=\operatorname{diag}[1,1,1,2,2,2]
$$

and express the tensorial norm of a stress-like quantity in the engineering notation as

$$
\|\boldsymbol{\sigma}\|_{\sigma}=\sqrt{\boldsymbol{\sigma}^{T} \boldsymbol{P} \boldsymbol{\sigma}}
$$

When transcribing the double contraction of two strain-like tensors, it is also necessary to insert a scaling matrix, but not the same one as for two stress-like tensors. Since the shear components have already been doubled, the corresponding scaling factors are now $1 / 2$ instead of 2 . So it turns out that the appropriate scaling matrix is the inverse of $\boldsymbol{P}$ and the tensorial norm of $\varepsilon$ is in the engineering notation evaluated as $\|\varepsilon\|_{\varepsilon}=\sqrt{\varepsilon^{T} \boldsymbol{P}^{-1} \varepsilon}$.

For the purpose of volumetric-deviatoric decomposition we need to establish the engineering counterpart of the unit second-order tensor (Kronecker delta). This is the tensor corresponding to the unit hydrostatic state, represented by a column matrix

$$
\boldsymbol{\delta}=\{1,1,1,0,0,0\}^{T}
$$

The volumetric-deviatoric decomposition is in the engineering notation based on projection matrices $\boldsymbol{I}_{V}=\frac{1}{3} \boldsymbol{\delta} \boldsymbol{\delta}^{T}$ and $\boldsymbol{I}_{D}=\boldsymbol{I}-\boldsymbol{I}_{V}=\boldsymbol{I}-\frac{1}{3} \boldsymbol{\delta} \boldsymbol{\delta}^{T}$. However, in the engineering transcription of expressions [2] and [15] for the elastic stiffness, the symmetric fourth-order unit tensor $\boldsymbol{I}_{S}$ must be replaced by the inverse scaling matrix $\boldsymbol{P}^{-1}$ (and not simply by the unit matrix $\boldsymbol{I}$ ):

$$
\boldsymbol{D}_{e}=3 \lambda \boldsymbol{I}_{V}+2 \mu \boldsymbol{P}^{-1}=3 K \boldsymbol{I}_{V}+2 G\left(\boldsymbol{P}^{-1}-\boldsymbol{I}_{V}\right)
$$

The generalized Hooke's law written separately for the volumetric and deviatoric part then reads

$$
\begin{aligned}
\sigma_{V} & =3 K \varepsilon_{V} \\
\boldsymbol{s} & =2 G \boldsymbol{P}^{-1} \boldsymbol{e}
\end{aligned}
$$




\section{Boundary value problem of linear elasticity}

If we restrict attention to sufficiently small changes of the initial configuration, a deformable body occupying a spatial domain $V$ can be described by the following equations:

$$
\begin{array}{rll}
\varepsilon_{i j}=\frac{1}{2}\left(\frac{\partial u_{i}}{\partial x_{j}}+\frac{\partial u_{j}}{\partial x_{i}}\right) & \ldots & \text { strain-displacement (kinematic) eqs. } \\
\sigma_{i j}=D_{i j k l}^{e} \varepsilon_{k l} & \ldots & \text { stress-strain (constitutive) equations } \\
\frac{\partial \sigma_{i j}}{\partial x_{j}}+\bar{b}_{i}=0 & \ldots & \text { equilibrium (static) equations }
\end{array}
$$

In engineering notation, these equations read

$$
\begin{aligned}
\boldsymbol{\varepsilon} & =\boldsymbol{\partial} \boldsymbol{u} \\
\boldsymbol{\sigma} & =\boldsymbol{D}_{e} \boldsymbol{\varepsilon} \\
\boldsymbol{\partial}^{T} \boldsymbol{\sigma}+\overline{\boldsymbol{b}} & =\boldsymbol{o}
\end{aligned}
$$

In the above, $\boldsymbol{u}=\left\{u_{1}, u_{2}, u_{3}\right\}^{T}$ is the displacement vector, $\boldsymbol{\varepsilon}$ is the column matrix of engineering strain components, $\boldsymbol{\sigma}$ is the column matrix of stress components, $\overline{\boldsymbol{b}}=$ $\left\{\bar{b}_{1}, \bar{b}_{2}, \bar{b}_{3}\right\}^{T}$ is the vector of body forces,

$$
\boldsymbol{\partial}=\left[\begin{array}{ccc}
\frac{\partial}{\partial x_{1}} & 0 & 0 \\
0 & \frac{\partial}{\partial x_{2}} & 0 \\
0 & 0 & \frac{\partial}{\partial x_{3}} \\
0 & \frac{\partial}{\partial x_{3}} & \frac{\partial}{\partial x_{2}} \\
\frac{\partial}{\partial x_{3}} & 0 & \frac{\partial}{\partial x_{1}} \\
\frac{\partial}{\partial x_{2}} & \frac{\partial}{\partial x_{1}} & 0
\end{array}\right]
$$

is an operator matrix that represents the engineering-notation counterpart of the symmetric gradient operator from [28], and $\boldsymbol{D}_{e}$ is the elastic material stiffness matrix, for isotropic materials given by [25].

The basic equations must be supplemented by appropriate boundary conditions,

$$
\begin{array}{rlll}
u_{i} & =\bar{u}_{i} \text { on } S_{u} & \ldots & \text { essential (kinematic) boundary conditions } \\
\sigma_{i j} n_{j} & =\bar{t}_{i} \text { on } S_{t} & \ldots & \text { natural (static) boundary conditions }
\end{array}
$$


or, equivalently,

$$
\begin{aligned}
\boldsymbol{u}=\overline{\boldsymbol{u}} & \text { on } S_{u} \\
\boldsymbol{n} \boldsymbol{\sigma}=\overline{\boldsymbol{t}} & \text { on } S_{t}
\end{aligned}
$$

where

$$
\boldsymbol{n}=\left[\begin{array}{cccccc}
n_{1} & 0 & 0 & 0 & n_{3} & n_{2} \\
0 & n_{2} & 0 & n_{3} & 0 & n_{1} \\
0 & 0 & n_{3} & n_{2} & n_{1} & 0
\end{array}\right]
$$

is a matrix containing the components $n_{i}$ of the unit outward normal to the boundary, and symbols with an overbar denote given quantities - the surface tractions $\overline{\boldsymbol{t}}=\left\{\bar{t}_{1}, \bar{t}_{2}, \bar{t}_{3}\right\}^{T}$ prescribed on a part $S_{t}$ of the boundary of $V$, and the displacements $\overline{\boldsymbol{u}}=\left\{\bar{u}_{1}, \bar{u}_{2}, \bar{u}_{3}\right\}^{T}$ prescribed on the remaining part $S_{u}$ of the boundary of $V$.

For problems formulated in a reduced space, e.g., under the assumptions of plane stress, plane strain, or axial symmetry, all matrices can be reduced accordingly.

\section{Discretization by finite elements}

In the standard displacement version of the finite element method (FEM), which is a generalization of the direct stiffness method of structural analysis, the displacement components are approximated as linear combinations of suitably chosen interpolation functions (in FEM called the shape functions) $N_{I}(\boldsymbol{x}), I=1,2, \ldots N_{\text {nod }}$. A typical property of the FE shape functions is that each of them is associated with one of $N_{\text {nod }}$ nodes of the model, and the value of the $I$-th shape function is equal to one at node number $I$ and equal to zero at all the other nodes. The displacement approximation reads

$$
u_{i}(\boldsymbol{x}) \approx \sum_{I=1}^{N_{\text {nod }}} N_{I}(\boldsymbol{x}) d_{I i}, \quad i=1,2,3
$$

where $d_{I i}$ are unknown displacement parameters. It is convenient to introduce matrix notation and collect the displacement parameters into a vector (column matrix) $\boldsymbol{d}$ and the shape functions into a matrix $N$. The approximation [40] is then rewritten as

$$
\boldsymbol{u}(\boldsymbol{x}) \approx \boldsymbol{N}(\boldsymbol{x}) \boldsymbol{d}
$$

The kinematic (strain-displacement) equations [31] provide an approximation of the strains,

$$
\varepsilon(x)=\partial u(x) \approx \partial N(x) d=B(x) d
$$


where $\boldsymbol{B}=\boldsymbol{\partial N}$ is the strain-displacement matrix (often called simply the B-matrix), containing the derivatives of the shape functions with respect to the spatial coordinates.

Substituting the strain approximation [42] into the constitutive equations [32] we obtain the stress approximation

$$
\boldsymbol{\sigma}(\boldsymbol{x}) \approx \boldsymbol{D}_{e}(\boldsymbol{x}) \boldsymbol{B}(\boldsymbol{x}) \boldsymbol{d}
$$

where the argument $\boldsymbol{x}$ at $\boldsymbol{D}_{e}$ marks explicitly that the elastic properties may be position-dependent.

It is clear that, by construction, the approximations of displacements, strains and stresses satisfy the kinematic and constitutive equations exactly. The static equations still need to be enforced. However, as the adopted approximations depend only on a finite number of unknown displacement parameters, the differential equations of equilibrium [33] in general cannot be satisfied exactly at every point of the body, i.e., in a strong sense. Instead of using these equations directly, we replace them by the principle of virtual work, i.e., by the requirement that the equality

$$
\int_{V} \boldsymbol{\sigma}^{T} \delta \varepsilon \mathrm{d} V=\int_{S_{t}} \overline{\boldsymbol{t}}^{T} \delta \boldsymbol{u} \mathrm{d} S+\int_{V} \overline{\boldsymbol{b}}^{T} \delta \boldsymbol{u} \mathrm{d} V
$$

must hold for an arbitrary virtual displacement field $\delta \boldsymbol{u}$ and virtual strain field $\delta \varepsilon$ satisfying the strain-displacement equations, $\delta \varepsilon=\boldsymbol{\partial} \delta \boldsymbol{u}$ in $V$, and the homogeneous kinematic boundary conditions, $\delta \boldsymbol{u}=\boldsymbol{o}$ on $S_{u}$. Equation [44] is the so-called weak form of the equilibrium equations. If the solution is sufficiently smooth, the strong form [33] and the weak form [44] are fully equivalent. However, when constructing equations for the displacement parameters we test the virtual work equality only for those virtual displacement fields (and the corresponding virtual strain fields) that have the same form as the approximate solution. In other words, we consider the virtual displacement as a linear combination of the shape functions,

$$
\delta \boldsymbol{u}(\boldsymbol{x})=\boldsymbol{N}(\boldsymbol{x}) \delta \boldsymbol{d}
$$

where $\delta \boldsymbol{d}$ is a column matrix of (arbitrary) virtual displacement parameters. The corresponding virtual strain field derived from the kinematic equations is then given by

$$
\delta \varepsilon(\boldsymbol{x})=\boldsymbol{B}(\boldsymbol{x}) \delta \boldsymbol{d}
$$

Substituting [43], [45] and [46] into the virtual work equality [44], we obtain the discretized weak form

$$
\begin{aligned}
\int_{V} \boldsymbol{d}^{T} \boldsymbol{B}^{T}(\boldsymbol{x}) \boldsymbol{D}_{e}^{T}(\boldsymbol{x}) \boldsymbol{B}(\boldsymbol{x}) \delta \boldsymbol{d} \mathrm{d} V= & \int_{S_{t}} \overline{\boldsymbol{t}}^{T} \boldsymbol{N}(\boldsymbol{x}) \delta \boldsymbol{d} \mathrm{d} S+ \\
& +\int_{V} \overline{\boldsymbol{b}}^{T} \boldsymbol{N}(\boldsymbol{x}) \delta \boldsymbol{d} \mathrm{d} V
\end{aligned}
$$


Taking into account that $\boldsymbol{d}$ and $\delta \boldsymbol{d}$ are not functions of the spatial coordinates and as such can be taken out of the integrals, we can rewrite [47] as

$$
\boldsymbol{d}^{T} \boldsymbol{K}_{e}^{T} \delta \boldsymbol{d}=\boldsymbol{f}_{e x t}^{T} \delta \boldsymbol{d}
$$

where

$$
\boldsymbol{K}_{e}=\int_{V} \boldsymbol{B}^{T}(\boldsymbol{x}) \boldsymbol{D}_{e}(\boldsymbol{x}) \boldsymbol{B}(\boldsymbol{x}) \mathrm{d} V
$$

is the (global) elastic stiffness matrix and

$$
\boldsymbol{f}_{\text {ext }}=\int_{S_{t}} \boldsymbol{N}^{T}(\boldsymbol{x}) \overline{\boldsymbol{t}} \mathrm{d} S+\int_{V} \boldsymbol{N}^{T}(\boldsymbol{x}) \overline{\boldsymbol{b}} \mathrm{d} V
$$

is the (equivalent) external force vector. Equation [48] is satisfied for an arbitrary column matrix of virtual displacement parameters $\delta \boldsymbol{d}$ if and only if

$$
\boldsymbol{K}_{e} \boldsymbol{d}=\boldsymbol{f}_{\text {ext }}
$$

These are the discretized equations of equilibrium from which it is possible to compute the unknown displacement parameters $\boldsymbol{d}$.

\section{Material nonlinearity}

The actual material behavior can be approximated as linear elastic only within a limited range of strains and strain rates. In general, the deformation processes taking place in real materials involve certain dissipative mechanisms, which lead to irreversible changes. This can be taken into account by refined models, which develop nonlinear stress-strain relations and consider the influence of the loading history. The current stress could be described by a functional of the previous strain history, but for numerical applications it is more convenient to replace the dependence on the entire history by the dependence on the current values of some additional internal variables characterizing the state of the material, such as the plastic strains in elastoplasticity.

Collecting all the internal variables into an object denoted as $\boldsymbol{\alpha}$ (scalar, tensor, or even a collection of scalars and tensors of various orders), we can formally write the general constitutive equations as

$$
\boldsymbol{\sigma}=\tilde{\boldsymbol{\sigma}}(\varepsilon, \boldsymbol{\alpha})
$$

where $\tilde{\sigma}$ denotes the constitutive operator. In the FEM, the stress approximation [43] is replaced by

$$
\boldsymbol{\sigma}(\boldsymbol{x}) \approx \tilde{\boldsymbol{\sigma}}(\boldsymbol{B}(\boldsymbol{x}) \boldsymbol{d}, \boldsymbol{\alpha}(\boldsymbol{x}))
$$

The discretized weak form of the equilibrium equations now reads

$$
\begin{aligned}
\int_{V} \tilde{\boldsymbol{\sigma}}^{T}(\boldsymbol{B}(\boldsymbol{x}) \boldsymbol{d}, \boldsymbol{\alpha}(\boldsymbol{x})) \boldsymbol{B}(\boldsymbol{x}) \delta \boldsymbol{d} \mathrm{d} V= & \int_{S_{t}} \overline{\boldsymbol{t}}^{T} \boldsymbol{N}(\boldsymbol{x}) \delta \boldsymbol{d} \mathrm{d} S+ \\
& +\int_{V} \overline{\boldsymbol{b}}^{T} \boldsymbol{N}(\boldsymbol{x}) \delta \boldsymbol{d} \mathrm{d} V
\end{aligned}
$$


or, equivalently,

$$
\boldsymbol{f}_{i n t}^{T}(\boldsymbol{d}, \boldsymbol{a}) \delta \boldsymbol{d}=\boldsymbol{f}_{\text {ext }}^{T} \delta \boldsymbol{d}
$$

where

$$
\boldsymbol{f}_{\text {int }}(\boldsymbol{d}, \boldsymbol{a})=\int_{V} \boldsymbol{B}^{T}(\boldsymbol{x}) \tilde{\boldsymbol{\sigma}}(\boldsymbol{B}(\boldsymbol{x}) \boldsymbol{d}, \boldsymbol{\alpha}(\boldsymbol{x})) \mathrm{d} V
$$

is the column matrix of (equivalent) internal forces, which now depend not only on the column matrix of displacement parameters $\boldsymbol{d}$ but also on the column matrix $\boldsymbol{a}$, which collects the values of internal variables $\boldsymbol{\alpha}$ at all material points that are used by the numerical integration scheme (the integral over the domain $V$ must now be approximated by a suitable numerical quadrature method). The final form of the discretized equations of equilibrium is then

$$
\boldsymbol{f}_{\text {int }}(\boldsymbol{d}, \boldsymbol{a})=\boldsymbol{f}_{\text {ext }}
$$

\section{Thermodynamic framework}

Since in general the internal variables evolve during the loading process, equations [57] must be supplemented by suitable equations governing the evolution of internal variables. Such evolution equations could be directly postulated in the form

$$
\dot{\boldsymbol{\alpha}}=\boldsymbol{g}(\dot{\varepsilon}, \varepsilon, \boldsymbol{\alpha})
$$

where $\boldsymbol{g}$ is a suitable function dependent on the current state of the material (reflected by the state variables $\varepsilon$ and $\alpha$ ) and on the imposed strain rate $\dot{\varepsilon}$.

To make sure that the material model is consistent with the second law of thermodynamics, it is important to check that the internal entropy production cannot be negative. For simplicity, we restrict attention to isothermal processes. As shown in Jirásek et al. (2002), Chapter 23, the second law in combination with the first law leads to the condition that there must exist a free energy potential $\psi(\varepsilon, \boldsymbol{\alpha})$ such that the quantity

$$
\mathcal{D}=\boldsymbol{\sigma}: \dot{\varepsilon}-\dot{\psi}
$$

is nonnegative for any admissible process. The difference $\mathcal{D}$ between the stress power $\boldsymbol{\sigma}: \dot{\boldsymbol{\varepsilon}}$ and rate of free energy $\dot{\psi}$ represents the dissipation rate per unit volume, in short referred to as the dissipation.

In the simplest case of an elastic material, the free energy potential depends only on the strain and no internal variables are needed. The dissipation can then be expressed as

$$
\mathcal{D}=\boldsymbol{\sigma}: \dot{\varepsilon}-\frac{\partial \psi}{\partial \varepsilon}: \dot{\varepsilon}=\left(\boldsymbol{\sigma}-\frac{\partial \psi}{\partial \varepsilon}\right): \dot{\varepsilon}
$$


Since individual components of the strain rate are independent and their signs are not constrained, the condition $\mathcal{D} \geq 0$ can be satisfied only if the term in the parentheses vanishes, i.e., if

$$
\boldsymbol{\sigma}(\varepsilon)=\frac{\partial \psi(\varepsilon)}{\partial \varepsilon}
$$

This is the stress-strain law, derived directly from the free energy potential.

The line of reasoning used in the previous paragraph is based on the tacit assumption that the stress depends on strain but not on the strain rate. This is indeed the case in standard elasticity. However, if one admits a special type of viscoelastic behavior with stress dependent on both the strain and the strain rate, equation [60] does not describe the total stress but only its part related to the reversible (elastic) deformation processes. An additional stress may arise from viscous effects and be dependent on the strain rate. In this case, we consider

$$
\boldsymbol{\sigma}_{Q}(\varepsilon)=\frac{\partial \psi(\varepsilon)}{\partial \varepsilon}
$$

as the conservative part of stress, and we can denote the difference $\boldsymbol{\sigma}-\boldsymbol{\sigma}_{Q}$ as the dissipative stress, $\sigma_{D}$, because the dissipation is according to [59] given by $D=$ $\sigma_{D}: \dot{\varepsilon}$. Instead of directly specifying the dependence of the dissipative stress on the strain rate, it is more convenient to postulate the existence of a dissipation potential $\phi$ and set, in analogy to [62],

$$
\boldsymbol{\sigma}_{D}(\dot{\varepsilon})=\frac{\partial \phi(\dot{\varepsilon})}{\partial \dot{\varepsilon}}
$$

The advantage is that the dissipation is then given by

$$
\mathcal{D}=\left(\boldsymbol{\sigma}-\frac{\partial \psi}{\partial \varepsilon}\right): \dot{\varepsilon}=\sigma_{D}: \dot{\varepsilon}=\frac{\partial \phi}{\partial \dot{\varepsilon}}: \dot{\varepsilon}
$$

and if the dissipation potential $\phi$ is convex and has its minimum at $\dot{\varepsilon}=\mathbf{0}$, then the dissipation is guaranteed to be nonnegative for any value of the strain rate $\dot{\varepsilon}$.

For models with internal variables, the free energy $\psi(\varepsilon, \boldsymbol{\alpha})$ depends on the strain and on internal variables. The dissipation evaluated as the difference between the stress power and the rate of internal energy is now

$$
\mathcal{D}=\boldsymbol{\sigma}: \dot{\boldsymbol{\varepsilon}}-\dot{\psi}=\left(\boldsymbol{\sigma}-\frac{\partial \psi}{\partial \varepsilon}\right): \dot{\boldsymbol{\varepsilon}}-\frac{\partial \psi}{\partial \boldsymbol{\alpha}}: \dot{\boldsymbol{\alpha}}=\left(\boldsymbol{\sigma}-\boldsymbol{\sigma}_{Q}\right): \dot{\boldsymbol{\varepsilon}}-\boldsymbol{\beta}_{Q}: \dot{\boldsymbol{\alpha}}
$$

where we have formally defined the (quasi)conservative thermodynamic forces conjugate to the internal variables as

$$
\boldsymbol{\beta}_{Q}(\varepsilon, \boldsymbol{\alpha})=\frac{\partial \psi(\varepsilon, \boldsymbol{\alpha})}{\partial \boldsymbol{\alpha}}
$$


The changes of internal variables are associated with certain dissipative processes (e.g. with plastic slip). It is therefore natural to assume that the dissipation can be expressed as

$$
\mathcal{D}=\frac{\partial \phi}{\partial \dot{\varepsilon}}: \dot{\varepsilon}+\frac{\partial \phi}{\partial \dot{\boldsymbol{\alpha}}}: \dot{\boldsymbol{\alpha}}=\boldsymbol{\sigma}_{D}: \dot{\boldsymbol{\varepsilon}}+\boldsymbol{\beta}_{D}: \dot{\boldsymbol{\alpha}}
$$

where

$$
\boldsymbol{\beta}_{D}=\frac{\partial \phi(\dot{\varepsilon}, \dot{\boldsymbol{\alpha}})}{\partial \dot{\boldsymbol{\alpha}}}
$$

are the dissipative thermodynamic forces conjugate to the internal variables. The dissipation potential $\phi(\dot{\boldsymbol{\varepsilon}}, \dot{\boldsymbol{\alpha}})$ is now a function of the rates of strain and of internal variables. It may also depend on the values of strain and internal variables, but this dependence has a secondary character.

We have established two expressions for the dissipation, given by [65] and [67], but they describe the same physical quantity and must give the same result for any combination of rates $\dot{\varepsilon}$ and $\dot{\boldsymbol{\alpha}}$. This leads to the identities $\boldsymbol{\sigma}-\boldsymbol{\sigma}_{Q}=\boldsymbol{\sigma}_{D}$ and $-\boldsymbol{\beta}_{Q}=$ $\boldsymbol{\beta}_{D}$. The first identity provides the constitutive law

$$
\boldsymbol{\sigma}=\boldsymbol{\sigma}_{Q}(\varepsilon, \boldsymbol{\alpha})+\boldsymbol{\sigma}_{D}(\dot{\varepsilon}, \dot{\boldsymbol{\alpha}})
$$

and the second is an implicit form of the evolution equation

$$
\boldsymbol{\beta}_{Q}(\varepsilon, \boldsymbol{\alpha})+\boldsymbol{\beta}_{D}(\dot{\varepsilon}, \dot{\boldsymbol{\alpha}})=0
$$

If [70] is solved for $\dot{\boldsymbol{\alpha}}$ as unknown, we obtain the explicit evolution equation in the form [58]. The advantage of the "implicit" approach based on the dissipation potential $\phi$ is that thermodynamic admissibility of the model is automatically ensured if $\phi$ is convex and has its minimum at $\dot{\varepsilon}=\mathbf{0}, \dot{\alpha}=\mathbf{0}$.

Conversion of the evolution equation to the explicit form can be based on the Legendre-Fenchel transform, which converts the dissipation potential into the socalled dual dissipation potential

$$
\phi^{*}\left(\boldsymbol{\sigma}_{D}, \boldsymbol{\beta}_{D}\right)=\max _{\dot{\varepsilon}, \dot{\boldsymbol{\alpha}}}\left(\boldsymbol{\sigma}_{D}: \dot{\varepsilon}+\boldsymbol{\beta}_{D}: \dot{\boldsymbol{\alpha}}-\phi(\dot{\boldsymbol{\varepsilon}}, \dot{\boldsymbol{\alpha}})\right)
$$

Based on this dual potential, the rate of internal variables can be expressed explicitly as

$$
\dot{\boldsymbol{\alpha}}=\frac{\partial \phi^{*}\left(\boldsymbol{\sigma}_{D}, \boldsymbol{\beta}_{D}\right)}{\partial \boldsymbol{\beta}_{D}}
$$

Dissipation potentials corresponding to viscous dissipative mechanisms are typically smooth and their derivatives are well defined in the classical sense. On the other hand, standard rate-independent plasticity or damage models give rise to non-smooth 
potentials. Fortunately, all presented equations remain valid if the derivatives are reinterpreted in the sense of subdifferentials; for more details see textbooks on convex analysis (Rockafellar, 1970) or Chapter 23 in Jirásek et al. (2002).

Material models with evolution equations derived from a dissipation potential are often referred to as generalized standard materials. The most popular example is the theory of plasticity with an associated flow rule. Such models comply with the postulate of maximum dissipation, which plays an essential role e.g. in the derivation of the fundamental theorems of classical plastic limit analysis. However, let us emphasize that the dissipation potential provides a convenient but relatively restricted framework for constitutive modeling. For instance, nonassociated flow rules may be more realistic for certain materials and, if formulated properly, they can still remain thermodynamically consistent. The dissipation inequality $\mathcal{D} \geq 0$ is easy to verify if the evolution equations are derived from a convex dissipation potential, but this does not mean that it is violated for all other models.

\section{Acknowledgements}

Financial support of the Ministry of Education of the Czech Republic under Research Plan MSM 6840770003 is gratefully acknowledged.

\section{References}

Jirásek M., Bažant Z. P., Inelastic Analysis of Structures, John Wiley and Sons, Chichester, 2002.

Rockafellar R. T., Convex Analysis, Princeton University Press, Princeton, NJ, 1970. 


\section{ANNEXE POUR LE SERVICE FABRICATION \\ A FOURNIR PAR LES AUTEURS AVEC UN EXEMPLAIRE PAPIER \\ DE LEUR ARTICLE ET LE COPYRIGHT SIGNE PAR COURRIER \\ LE FICHIER PDF CORRESPONDANT SERA ENVOYE PAR E-MAIL}

1. ARTICLE POUR LA REVUE :

REGC - 11/2007. Damage and fracture in geomaterials

2. Auteurs :

Milan Jirásek

3. TITRE DE L'ARTICLE :

Basic concepts and equations

of solid mechanics

4. TITRE ABRÉGÉ POUR LE HAUT DE PAGE MOINS DE 40 SIGNES :

Basic concepts and equations

5. DATE DE CETTE VERSiON :

September 17, 2007

6. CoORdonnéES DES AUTEURS :

- adresse postale :

Department of Mechanics

Faculty of Civil Engineering

Czech Technical University in Prague

16629 Prague, Czech Republic

Milan.Jirasek@epfl.ch

- téléphone : +420-224354481

- télécopie : +420-224310775

- e-mail : milan.jirasek@fsv.cvut.cz

7. LOGICIEL UTILISÉ POUR LA PRÉPARATION DE CET ARTICLE :

LATEX, avec le fichier de style article-hermes2.cls, version $1.23 \mathrm{du}$ 02/08/2006.

8. FORMULAIRE DE COPYRIGHT :

Retourner le formulaire de copyright signé par les auteurs, téléchargé sur :

http://www. revuesonline.com

SERVICE ÉditORIAL - HERMES-LAVOISIER 14 rue de Provigny, F-94236 Cachan cedex

Tél. : 01-47-40-67-67

E-mail : revues@lavoisier.fr

Serveur web : http://www.revuesonline.com 